\title{
Pre-Fibrotic Changes are Induced in the Guinea Pig Liver in Response to Cardiorespiratory, Central Nervous System, and Gastrointestinal Stressors
}

\section{$\underline{\text { Michael E. Motokata* }}$}

* To whom correspondence should be addressed: Harvard Medical School, 333 Vanderbilt Hall, Boston, MA 02115-5750, USA

\section{ABSTRACT}

To determine the relationship between stress and the incidence of liver fibrosis, 140 guinea pigs were exposed to various stressors, and their post-mortem livers were assessed. Four stress groupscardiorespiratory (CR), central nervous system (CNS), gastrointestinal (GI), and combined (Cd)were designated in accordance with the stressor(s) experienced and were compared to unstressed control subjects. By blood chemistry analysis, the most pervasive findings were decreased glucose and increased amylase. Stress group blood glucose levels ranged from $22 \%$ to $38 \%$ below that of nonstressed controls, and serum amylase was increased by $35 \%$ to $68 \%$ relative to controls. The reduction in glucose was significant in the CR and GI groups, and the elevation in amylase was significant in the CR, GI, and Cd groups. Pathologically, the most frequent finding among the four groups was fatty change, present in $44 \%$ of stressed subjects, followed by passive congestion, observed in $40 \%$. The Cd group demonstrated a significantly increased incidence of congestion, while both the $\mathrm{Cd}$ and GI groups showed a significantly increased incidence of fatty change. Subjects in whom congestion was detected showed a 1.7-fold greater fibroblast proliferation than subjects in whom fatty change was seen. The most extensive pathological changes were manifested in the $\mathrm{Cd}$ group, in the form of congestion, hemorrhage, fatty change, and fibroblast proliferation. Among the three single-stress groups, the greatest degree of fibroblast proliferation and collagen deposition, and hence the greatest potential for fibrosis, was evident in the GI group. The fibroblastosis in the GI group was statistically significant, presenting a direct pathological indication of pre-fibrotic change. These results provide preliminary evidence that stress is capable of inducing pathological processes in the liver that may lead to fibrosis and, ultimately, to cirrhosis.

\section{INTRODUCTION}

Liver fibrosis is a common morphological response to hepatocellular injury of diverse etiologies, including 
infection, toxins, inflammation, biliary obstruction, and vascular disturbance. Hepatitis B infection and chemical injury, such as that induced by carbon tetrachloride, are important causes of liver fibrosis. Ethanol has a direct toxic effect on the liver, and is responsible for significant morbidity and mortality. It is estimated that alcohol abuse accounts for $60-70 \%$ of cases of cirrhosis in the Western world (1).

The pathogenesis of fibrosis involves a series of cytokine-mediated events. Initial injury typically leads to hepatocellular necrosis, followed by inflammation, activation of macrophages, and the consequent elaboration of cytokines such as platelet-derived growth factor (PDGF), transforming growth factor-alpha (TGF-*) and -beta (TGF-B), tumor necrosis factor-alpha (TNF-*), and interleukin-1, -6, and -11 $(2,3)$. Cytokines released by immune cells during the inflammatory reaction lead to activation of fat-storing cells, increased production of extracellular matrix, and decreased matrix degradation (2). This process culminates in the net accumulation of matrix and concomitant destruction of normal liver architecture seen in fibrosis.

Because cytokines play such a prominent, albeit ill-defined, role in the pathogenesis of liver disease, an understanding of their regulation may provide insight into future treatments. Recent advances in the field of neuroendocrinimmunology have shown that stress, whether physical, chemical, or psychosocial, interferes with the function of the immune system (4), ultimately affecting cytokine regulation. However, relatively little research has specifically addressed the potential role of stress in hepatic dysfunction.

The objective of this study was to determine whether stress can induce liver fibrosis and cirrhosis and, more specifically, which type of stressor appears most damaging. Because cirrhosis is a chronic process typically developing over a period of years, this study focuses primarily on the detection of the early more acute pathological changes in the liver, including fibroblastosis and collagen deposition, the two pathological criteria for fibrosis.

\section{MATERIALS AND METHODS}

\section{Experimental Design}

Three stressors were utilized in this study. Cardiorespiratory stress was induced by forced exercise, CNS stress by sleep deprivation and rotational motion, and gastrointestinal stress by reduced access to food, provided at irregular intervals. Male guinea pigs of the Hartley strain (600-1200g body weight) were divided into five study groups: cardiorespiratory stress (CR) group, CNS stress (CNS) group, gastrointestinal stress (GI) group, combined stress (Cd) group, and unstressed control group. The Cd group was exposed to all three individual stressors: cardiorespiratory, central nervous system, and gastrointestinal. After one week of acclimation to their new environment, the subjects were studied for three weeks according to the schedule shown in Table 1. The CR stressor consisted of 15 min of forced exercise on a treadmill set at $6 \mathrm{mph}$, which appeared to induce the maximum tolerable level of excercise in the guinea pigs. In terms of CNS stressors, sleep deprivation was produced by placing bright lights at close proximity to their cages overnight, and rotational motion was generated by placing the animals in a rotating raffle cylinder.

Throughout the experiment, the guinea pigs were kept in an outdoor laboratory environment with the temperature ranging from $68-93^{\circ} \mathrm{F}$. Except for the GI and Cd groups, the animals were provided unlimited access to pellet feed and, in all cases, to water. All animals were ethically and humanely treated in accordance with the "Guide for Care and Use of Laboratory Animals" (National Institutes of Health, Bethesda, MD, USA).

\section{Blood Analysis}

At the end of the study, an average of $4 \mathrm{~mL}$ of blood was immediately drawn from the heart of each sacrificed guinea pig and analyzed for glucose, total protein, albumin, globulin, ALT, AST, amylase, CPK, and LDH 
levels (Sim Clinical Laboratory, Manila, Philippines).

\section{Pathological Analysis}

Excised livers were completely sectioned, and two to four sections were randomly selected and routinely stained with hematoxylin and eosin. Sections were examined for the presence of pathology, with particular emphasis on the following features: congestion, hemorrhage, fatty change, inflammation, necrosis, and collagen deposition. Specimens showing evidence of a specific pathological feature in any of the selected sections were considered as positive for that feature. In the case of collagen deposition, free-standing loose fibers were considered artifactual. Fibroblast counts were also performed; three lobular areas with no visible portal fibrous change were randomly selected, and the number of fibroblasts within six high-power (400x) fields was counted and subsequently averaged. All sections were reviewed by a staff pathologist for confirmation.

\section{Statistical Analysis}

Blood chemistry data were evaluated by two-tailed Student's $t$-test to determine whether the analyte levels in each stress group varied significantly from control values. In addition, two-sided Chi-square analysis was used to detect significant differences between stress and control groups with respect to the prevalence of each aforementioned histopathological finding, and two-tailed Student's $t$-test was used to discern any statistically significant variation in fibroblast count between stress and control groups.

\section{RESULTS}

The blood chemistry values for the 11 analytes measured among the five study groups are presented in Table 2. In the CR and GI groups, glucose levels were significantly ( $p<0.01$ and $p<0.05$, respectively) decreased in comparison to levels obtained from the unstressed control group. In addition, a significant $(p<0.005)$ diminution in total serum protein was detected in the CNS group. Serum albumin was significantly $(p<0.05)$ lower than control values in the case of the CR group, and globulin was significantly $(p<0.002)$ diminished in the CNS group. In terms of liver enzymes, a significant $(p<0.05)$ elevation in the AST/ALT ratio was noted in the Cd group, and a significant $(p<0.001)$ rise in ALT was found in the CR group. Furthermore, in the Cd, CR, and GI groups, amylase was significantly ( $p<0.05, p<0.001$, and $p<0.05$, respectively) increased in comparison to the control group.

On gross pathological examination of the excised livers, approximately $80 \%$ showed gallbladder distension, which appeared more frequently in the GI group than the three remaining stress groups or the control group. In many cases, the livers were firm and showed yellow discoloration, with numerous fissures on their surfaces. By microscopy, pathological changes ranged from focal to panlobular, and from early lesions to those in late necrotic stages. Many of the classical signs of necrosis were noted in the stress groups, both stage I (e.g., cellular edema, detachment of ribosomes) and stage II (e.g., vacuolization of mitochondria, membrane damage, lysosomal degradation). In addition, atypical proliferation of bile ductules, parenchymal destruction, luminal narrowing of bile ductules, fibrous obliteration of intrahepatic bile ductules, and piecemeal necrosis of varying degrees were apparent. In the parenchyma, hepatocyte cords ranged from intact to disorganized, and many sinusoids were dilated. Edema was occasionally present, some of which appeared in the bile ductules. Cholestasis, however, was not commonly observed.

The results of histopathological examination of the excised livers are presented in Table 3. A significant $(p<$ 0.025 ) increase in the proportion of subjects whose livers showed congestion was detected in the Cd group. No statistically significant variations in the number of specimens showing hemorrhage, inflammation, or collagen deposition were detected in the four stress groups. In those specimens demonstrating collagen deposition, the change occurred primarily in portal and periportal areas, with sparse evidence in perivenular 
regions (Fig. 1). Extensive fibrosis frequently led to occlusion of portal vessels and bile ductules. Accordingly, tortuosity of some portal vessels and bile ductules was noted.

Prevalence of necrosis among liver specimens was not statistically significant among any of the stress groups. Where apparent, necrotic lesions were primarily of the coagulation type, and often appeared in and around the fatty change lesions. Numerous sections showed a nutmeg pattern. In the necrotic regions, many of the cell membranes were ruptured, cellular contents were frequently inapparent, and nuclear karyorrhexis and vacuolation were abundant.

In the $\mathrm{Cd}$ and GI groups, there was a significant increase in the incidence of fatty change $(p<0.001$ and $p<$ 0.05 , respectively). The fatty change was predominantly centrilobular, becoming progressively less apparent toward the periphery. In these lesions, hydropic degeneration of hepatocytes was frequently observed. The fibroblast counts revealed a significant $(p<0.005)$ fibroblastosis in the GI group and a significant $(p<0.05)$ decrease in fibroblast proliferation in the CNS group. In all groups, fibroblasts were found mostly in the space of Disse and less frequently in the sinusoids (Fig. 2), but were rarely present in regions of necrosis or fatty change.

\section{DISCUSSION}

The results of blood chemistry analysis reveal three principal statistically significant changes among the four stress groups in comparison to the unstressed control groups. These deviations were decreased glucose, elevated amylase, and reduced albumin, globulin, and total protein.

Decreased blood glucose was present in all four stress groups, each of which also demonstrated an increase in the incidence of fatty change relative to the control group. In fact, the most marked pathological finding among the stress groups was fatty change, which was significant in the GI and Cd groups. The occasional association of decreased blood glucose and fatty change among stress subjects is likely to be a consequence of the increase in lipolysis, free fatty acid (FFA) entry into the liver, and subsequent reesterification to triglycerides that occurs in response to hypoglycemia. It has been shown that 60 hours of fasting can induce a 2.5-fold increase in FFA reesterification (5). This increased reesterification results in lipid accumulation in the liver, leading to fatty change. It should be noted, however, that the results in this experiment do not show a strong correlation between fatty change and decreased blood glucose; for example, the Cd group shows a mild reduction in glucose yet the highest frequency of fatty change. This finding suggests that other important factors may be functional in the induction of fatty change.

Fatty change, whether produced by hypoglycemia or through another mechanism, often precedes liver fibrosis. It is thought that the inflammatory process associated with fatty change is ultimately responsible for the fibrosis, via the action of mobilized and stimulated Kupffer cells (2). Investigations have shown that activated Kupffer cells, through their production of cytokine mediators such as TGF- $\beta$, stimulate the proliferation of hepatic lipocytes $(6,7)$ and their synthesis of collagen $(8)$, proteoglycans $(9,10)$, and hyaluronate (11). Hepatic lipocytes have a high proliferative capacity, and are capable of transforming to fibroblast-like cells $(12,13)$. The lipocytes and, to a greater degree, the transformed cells produce significant quantities of connective tissue components similar to those found in the fibrotic extracellular matrix (14). However, the results of pathological analysis in this study do not support the paradigm of fibrosis secondary to fatty change. When fibroblastosis was evident on pathological examination, the fibroblasts appeared mostly in the space of Disse and sinusoids, and only infrequently in areas of fatty change. In areas of intense fatty change, marked necrosis, but few fibroblasts were observed.

The second major blood chemistry result among the stress groups was a significant reduction in albumin, globulin, and total protein. In states of hypoproteinemia, the synthesis of cellular proteins, such as the apolipoprotein B component of VLDL and LDL, is limited $(15,16)$. Through their role in triglyceride 
transport and cellular uptake of FFAs and cholesterol, these proteins are crucial to lipid metabolism. Thus, like hypoglycemia, hypoproteinemia may result in lipid accumulation in the liver, leading to fatty change and, thereby, to fibrosis.

It is noteworthy that a mild level of fatty change was observed in the control group. This may be interpreted either as an incidental finding or as an indication that, in addition to those stressors tested in the experiment, secondary stressors may have been operative. Particularly relevant are stressors such as fear and cold. Despite attempts to control for extraneous stressors, the short duration of the study may not have allowed for sufficient acclimation to the experimental environment. It was noted that, whether from fear or cold, the subjects tended to crouch in the corners of their cages and cluster on top of one another. Fear and cold are known to induce ACTH (17), a major mediator of the stress response, and to raise steroid hormone output by the adrenal cortex. Nevertheless, any background level of stress that may have contributed to liver changes was eliminated from the interpretation of results, as stress group blood chemistry and histopathological data were statistically analyzed with respect to those of the control group.

The third principal blood chemistry result among the stress groups was a significant increase in amylase with respect to control values. By blood chemistry analysis, elevations in AST, ALT, and amylase are indicators of liver damage $(18,19)$ that, if prolonged, can lead to fibrosis. These increases are non-specific, and in the context of this experiment, may stem from chronic passive congestion (CPC), inflammation, and fatty change, all of which produce hepatocyte injury and necrosis, with consequent leakage of ALT and AST into the circulation. The elevated AST in the GI group, albeit statistically insignificant, may be related to diminished clearance of AST, a function of sinusoidal cells that is easily disrupted in the development of fibrosis (20). Extrahepatic biliary obstruction is a common cause of altered liver function indices, but is an unlikely cause in this study due to the sparse evidence of cholestasis on pathological investigation. Although no significant AST and ALT elevations were observed, amylase was significantly elevated in the Cd, CR, and GI stress groups.

The significant rise in amylase seen in all stress groups other than the CNS group may have several causes. Sympathetic excitation induced by stress hormones elicits vasospasm and possible thrombosis. The resultant hypoxia is thought to produce ischemic injury of hepatocytes and pancreatic acinar cells, manifested by elevated serum amylase (21). In prolonged stress states, chronic pancreatitis can result $(22,23)$, and the inflammatory process is capable of producing extensive fibrosis. An alternative mechanism for the increased amylase levels is acute liver congestion early in the progression of cardiac circulatory failure (24).

CPC, through hepatocyte damage, may also be implicated in the rise in serum amylase. CPC may arise from hypertension mediated by chronic elevations in catecholamines during prolonged stress. Whether directly through ischemia or indirectly through amylase-mediated destruction, the hepatocyte injury resulting from CPC resolves by organization and centrilobular fibrosis (25). This mechanism is supported by the pathology results of this study, which suggest that congestion predisposes to fibrosis more significantly than does fatty change. Twenty-six percent of subjects in whom congestion was detected showed evidence of collagen deposition, as compared to $15 \%$ of subjects in whom fatty change was detected.

Of the three single-stress groups examined, CR and GI appear most predisposed to liver fibrosis based on blood chemistry analysis, in particular with respect to serum amylase. However, only in the GI and Cd groups was the elevated amylase positively correlated with pathological changes in the liver. Of the two pathological criteria for fibrosis, the most direct is collagen deposition, although this is a chronic rather than acute change. While the Cd group demonstrated the most prevalent and extensive collagen deposition, this finding was not statistically significant. Nevertheless, in the GI group there was significant evidence of fibroblastosis, the second pathological criterion for fibrosis.

The fact that the significant fibroblastosis detected in the GI group was not found in the Cd group is rather 
enigmatic. Furthermore, it is surprising that the CNS group exhibited a decrease in fibroblast count rather than an increase or no change. These findings raise the possibility that the CNS stressor provokes a unique anti-fibrotic response. If this is the case, then the absence of significant change in the fibroblast count of the Cd group may represent the net effect of opposing fibroblast responses to the CNS and GI stressors.

A few qualifications must be made with regard to the results of this study. Although the blood chemistry and pathology findings indicate that the GI stress induces the most significant pre-fibrotic changes, comparison of the effects of different stressors must be undertaken with caution. Secondly, these stressors were modeled through experimental methods in the guinea pig and, as such, may not directly reflect the experience of these stressors in humans. Interestingly, it has been shown that humans exhibit a far more exuberant collagen response to injury than do other animals, including other primates (26). In addition, it should be noted that the observed blood chemistry values of the control group in several cases differed considerably from normal values, in the direction of stress group values. This difference may have led to an underestimation of the significance of the stress group results, as blood chemistry and histopathological data were statistically analyzed with respect to those of the control group rather than the accepted normal values. Thirdly, the mortality of several animals over the course of the experiment led to their exclusion from the data pools in order to reduce the possibility for error introduced by extraneous factors, such as underlying illness. In most cases, however, autopsy of these animals revealed acute liver damage. In this respect, the significance of the study's findings, again, are likely to have been understated.

In conclusion, this study provides preliminary evidence that prolonged stress is capable of eliciting pathological changes in the liver that may lead to fibrosis and, ultimately, to cirrhosis. The statistical significance of changes in blood chemistry as well as the prevalence of pathological changes may be enhanced in future studies utilizing a larger sample population. In addition, subsequent investigations of longer duration are needed to correlate the finding of fibroblastosis in this investigation with definitive pathological observations of collagen deposition. Future studies may incorporate recently proposed biochemical markers for fibrosis, including metalloproteinases, oxyradicals, glutathione (27-29), LDH, and membrane lipids (30). If the preliminary findings in this study are corroborated by evidence from further investigations, preventive medicine will require a new emphasis on various forms of stress, particularly GI stress, as potential risk factors for the development of liver fibrosis and cirrhosis.

\section{ACKNOWLEDGMENTS}

The author is indebted to Juliet Santos and Judaz Ogawa, M.D. for provision of lab space, materials, and financial support. Much credit is also due to Erniel Barrios, Ph.D. and Remedios Magkasi, M.D. for their assitance in statistical and pathological analysis, respectively.

\section{REFERENCES}

1. Cotran RS, Kumar V, Robbins SL. Robbins Pathologic Basis of Disease. 5th ed. Philadelphia: WB Saunders Co.; 1994: 834.

2. Gressner AM, Bachem MG. Cellular communications and cell-matrix interactions in the pathogenesis of fibroproliferative diseases: liver fibrosis as a paradigm. Annales de Biologie Clinique 52(3): 205-226; 1994.

3. Andus T, Holstege A. Cytokines and the liver in health and disease. Effects on liver metabolism and fibrogenesis. Acta Gastroenterologica Belgica 57(3-4): 236-244; 1994.

4. Khansari DN, Murgo AJ, Faith RE. Effects of stress on the immune system. Immunology Today 11(5): $170-175 ; 1990$.

5. Carlson MG, Snead WL, Campbell PJ. Fuel and energy metabolism in fasting humans. American Journal 
of Clinical Nutrition 60(1): 29-36; 1994.

6. Friedman SL, Arthur MJ. Activation of cultured rat hepatic lipocytes by Kupffer cell conditioned medium. Direct enhancement of matrix synthesis and stimulation of cell proliferation via induction of platelet-derived growth factor receptors. Journal of Clinical Investigation 84: 1780-1785; 1989.

7. Zerbe O, Gressner AM. Proliferation of fat-storing cells is stimulated by secretion of Kupffer cells from normal and injured liver. Experimental and Molecular Pathology 49(1): 87-101; 1988.

8. Matsuoka M, Pham NT, Tsukamoto H. Differential effects of interleukin-1-alpha, tumor necrosis factor alpha, and transforming growth factor-beta-1 on cell proliferation and collagen formation by cultured fatstoring cells. Liver 9(2): 71-78; 1989.

9. Meyer DH, Bachem MG, Gressner AM. Modulation of hepatic lipocyte proteoglycan synthesis and proliferation by Kupffer cell-derived transforming growth factors type beta-1 and type alpha. Biochemical and Biophysical Research Communications 171(3): 1122-1129; 1990.

10. Gressner AM, Zerbe O. Kupffer cell-mediated induction of synthesis and secretion of proteoglycans by rat liver fat-storing cells in culture. Journal of Hepatology 5(3): 299-310; 1987.

11. Gressner AM, Haarmann R. Regulation of hyaluronate synthesis in rat liver fat storing cell cultures by Kupffer cells. Journal of Hepatology 7(3): 310-318; 1987.

12. Mak KM, Leo MA, Lieber CS. Alcoholic liver injury in baboons: transformation of lipocytes to transitional cells. Gastroenterology 87(1): 188-200; 1984.

13. McGee JO, Patrick RS. The role of perisinusoidal cells in hepatic fibrogenesis. An electron microscopic study of acute carbon tetrachloride liver injury. Laboratory Investigation 26(4): 429-440; 1972.

14. Davis BH. Transforming growth factor beta responsiveness is modulated by the extracellular collagen matrix during hepatic ito cell culture. Journal of Cellular Physiology 136(3): 547-553; 1988.

15. Bouziane M, Prost J, Belleville J. Dietary protein deficiency affect n-3 and n-6 polyunsaturated fatty acids hepatic storage and very low density lipoprotein transport in rats on different diets. Lipids 29(4): 265-272; 1994.

16. Bullock BL, Rosendahl PP. Pathophysiology: adaptations and alterations in function. 3rd ed. Philadelphia: J.B Lippincott Co.; 1992.

17. Sasaki F, Wu P, Rougeau D, et al. Cytochemical studies of responses of corticotropes and thyrotropes to cold and novel environment stress. Endocrinology 127(1): 285-297; 1990.

18. Chopra S, Griffin PH. Laboratory tests and diagnostic procedures in evaluation of liver disease. American Journal of Medicine 79(2): 221-230; 1985.

19. Del Olmo Martinez ML, Barba Bermejo M. [Changes in pancreatic secretion in alcoholic liver disease.] Revista Espanola de Enfermedades Digestivas 77(3): 197-204; 1990.

20. Williams AL, Hoofnagle JH. Ratio of serum aspartate to alanine aminotransferase in chronic hepatitis . Relationship to cirrhosis. Gastroenterology 95(3): 734-739; 1988.

21. Yamaguchi H, Kimura T, Nawata H. Does stress play a role in the development of severe pancreatitis in 
rats? Gastroenterology 98(6): 1682-1688; 1990.

22. Tanaka T, Ichiba Y, Miura Y. et al. Canine model of chronic pancreatitis due to chronic ischemia. Digestion 55(2): 86-89; 1994.

23. Freiburghaus AU, Redha F, Ammann RW. Does acute pancreatitis progress to chronic pancreatitis? A microvascular pancreatitis model in the rat. Pancreas 11(4): 374-381; 1995.

24. Goth L, Meszadros I, Scheller G. Hyperamylasemia and alpha-amylase isozymes in acute liver congestion due to cardiac circulatory failure. Clinical Chemistry 35(8): 1793-1794; 1989.

25. Shibayama Y, Urano T, Asaka S, et al. Pathogenesis of centrilobular necrosis following congestion of the liver. Journal of Gastroenterology \& Hepatology 8(6): 530-534; 1993.

26. Kissane JM, ed. Anderson's Pathology. 8th ed. St. Louis: CV Mosby Co.; 1985: 1106.

27. Irita K, Okabe H, Koga A, et al. Increased sinusoidal efflux of reduced and oxidized glutathione in rats with endotoxin/D-galactosamine hepatitis. Circulatory Shock 42(3): 115-120; 1994.

28. Schoenberg MH, Buchler M. Pietrzk C, et al. Lipid peroxidation and glutathione metabolism in chronic pancreatitis. Pancreas 10(1): 36-43; 1995.

29. Luthen R, Niederau C, Grendell JH. Intrapancreatic zymogen activation and levels of ATP and glutathione during caerulein pancreatitis in rats. American Journal of Physiology 268(4 pt 1): G592-G604; 1995.

30. Takayama F, Egashira T, Yamanaka Y. The multiple hydroperoxides of choline phospholipids occuring in plasma after ischemia-reperfusion in rat liver. Journal of Toxicological Sciences 19(2): 97-106; 1994.

\section{BIOGRAPHY}

Michael E. Motokata is presently a fourth-year medical student at Harvard Medical School (Boston, Massachussetts, USA). He received his B.A. from the University of Minnesota in 1982. Prior to medical school, the author worked as an investment banker on Wall Street, which inspired his research on the clinical impact of stress. The author initiated this study during his second year at the Fatima College of Medicine (Manila, Philippines) and completed it as a third-year student at Harvard Medical School. Upon graduation, he will pursue a residency position in Obstetrics/Gynecology.

Copyright (C) 1996 by MJM 